\title{
Comparison of the NIST and NPL Air Kerma Standards Used for X-Ray Measurements Between $10 \mathrm{kV}$ and $80 \mathrm{kV}$
}

\section{O'Brien and P. Lamperti}

National Institute of Standards and Technology,

Gaithersburg, MD 20899-8460, USA

michelle.obrien@nist.gov paul.lamperti@nist.gov

and

A direct comparison was made between the air kerma primary standards used for the measurements of low-energy $\mathrm{x}$ rays at the National Institute of Standards and Technology (NIST) and the National Physical Laboratory (NPL). The comparison was conducted at the NPL using NPL reference radiation qualities between $10 \mathrm{kV}$ and $80 \mathrm{kV}$. The results show the primary airkerma standards to agree within $0.6 \%$ of their values for beam qualities up to $80 \mathrm{kV}$.
Key words: air kerma; free-air ionization chamber; primary standard; reference radiation qualities.

Accepted: July 28, 2000

Available online: http://www.nist.gov/jres

\section{T. Williams and T. Sander}

National Physical Laboratory, Teddington, Middlesex, UK

\section{Introduction}

A direct comparison was made between the air kerma primary standards used for the measurements of lowenergy $x$ rays at the National Institute of Standards and Technology (NIST) and the National Physical Laboratory (NPL). The comparison was conducted in June 1998 at the NPL using NPL tungsten reference radiation qualities between $10 \mathrm{kV}$ and $80 \mathrm{kV}$ and the new mammography $28 \mathrm{kV}$ entrance and exit beam offered at NPL. The two NIST primary standards shipped to the NPL for this comparison were the Lamperti $(10 \mathrm{kV}$ to $60 \mathrm{kV})$ and the Ritz $(20 \mathrm{kV}$ to $100 \mathrm{kV})$ free-air ionization chambers. Prior to this comparison these primary $\mathrm{x}$-ray standards at these energies have only been compared indirectly through comparisons at the Bureau nternational des Poids et Mesures (BIPM).

\section{NPL Irradiation Facilities}

Two x-ray irradiation laboratories at NPL were used for this comparison. A constant-potential low-ripple generator connected to Machlett ${ }^{1}$ OEG-50A x-ray tubes with either a tungsten or molybdenum anode, each having $1 \mathrm{~mm}$ of beryllium inherent filtration, is used to perform calibrations in the NPL low-energy x-ray labo ratory. The $\mathrm{x}$-ray tube voltage may be varied from $8 \mathrm{kV}$

\footnotetext{
${ }^{1}$ Certain commercial equipment, instruments, or materials are identified in this paper to foster understanding. Such identification does not imply recommendation or endorsement by the National Institute of Standards and Technology, nor does it imply that the materials or equipment identified are necessarily the best available for the purpose.
} 
to $50 \mathrm{kV}$ in $0.1 \mathrm{kV}$ steps and the tube current is adjustable from $10 \mu \mathrm{A}$ to $17 \mathrm{~mA}$ in $10 \mathrm{~mA}$ steps. In the medium-energy $\mathrm{x}$-ray laboratory a constant-potential low-ripple generator connected to either a Philips $160 \mathrm{kV} \mathrm{x}$-ray tube with an inherent filtration of $1 \mathrm{~mm}$ beryllium, or a Muller $300 \mathrm{kV}$ tube with an inherent filtration equivalent to $4 \mathrm{~mm}$ aluminum, is used to perform calibrations. The voltage may be varied from $50 \mathrm{kV}$ to $300 \mathrm{kV}$ and the current is adjustable from $10 \mu \mathrm{A}$ to $25 \mathrm{~mA}$. The output of each $\mathrm{x}$-ray system was measured through the use of a transmission monitor chamber. All charge measurements were normalized to the response of the monitor chamber. In the low-energy facility the comparison measurements were made at a distance from the $\mathrm{x}$-ray focal spot of $0.5 \mathrm{~m}$ with a field size of $40 \mathrm{~mm}$ diameter at the point of measurement. In the medium-energy facility the comparison measurements were made at a distance from the $\mathrm{x}$-ray focal spot of $0.75 \mathrm{~m}$ with a field size of $63 \mathrm{~mm}$ diameter at the point of measurement. The x-ray beams produced in both NPL calibration facilities are sufficiently uniform to perform primary standard comparisons and calibrations. The NPL reference radiation qualities used for the comparison are listed in Table 1.

\section{Determination of the Air Kerma Rate}

The air kerma rate is determined by the relationship

$$
K=\left(\frac{I}{m}\right)\left(\frac{W}{e}\right)(1-g)^{-1} \Pi k_{i},
$$

Table 1. The NPL reference radiation qualities used for the comparison

\begin{tabular}{lcccc}
\hline \hline $\begin{array}{c}\text { NPL reference } \\
\text { number }\end{array}$ & $\begin{array}{c}\text { Generating } \\
\text { potential } \\
(\mathrm{kV})\end{array}$ & $\begin{array}{c}\text { Half-value } \\
\text { layer } \\
(\mathrm{mm} \text { A1 })\end{array}$ & A1 filtration & $\begin{array}{c}\text { Air kerma } \\
\text { rate } \\
\left(\mathrm{mGy} \mathrm{s}^{-1}\right)\end{array}$ \\
\hline \multicolumn{5}{c}{ Tungsten anode } \\
\hline 2.4 .2 & 10 & 0.036 & 0.025 & 2.1 \\
2.4 .3 & 11.5 & 0.050 & 0.050 & 2.5 \\
2.4 .4 & 14 & 0.07 & 0.11 & 2.2 \\
2.4 .5 & 16 & 0.10 & 0.20 & 2.5 \\
2.4 .6 & 20 & 0.15 & 0.30 & 3.0 \\
2.4 .7 & 24 & 0.25 & 0.45 & 2.2 \\
2.4 .8 & 34 & 0.35 & 0.47 & 3.7 \\
2.4 .9 & 41 & 0.50 & 0.56 & 4.1 \\
2.4 .10 & 44 & 0.70 & 0.74 & 3.7 \\
2.4 .11 & 50 & 1.00 & 1.01 & 3.1 \\
2.2 .1 & 50 & 1.00 & 0.75 & 1.5 \\
RQR6 & 80 & 2.9 & 2.7 & 1.3 \\
\hline \multicolumn{5}{c}{ Molybdenum Anode } \\
\hline Entrance & 28 & 0.30 & 0.03 Mo & 0.6 \\
Exit & 28 & 0.62 & 0.03 Mo & 0.02 \\
& \multicolumn{5}{c}{+ breast phantom } \\
\hline
\end{tabular}

where

$I / m$ is the mass ionization current as measured by the free-air ionization chamber,

W/e is the mean energy per unit charge expended by electrons in dry air with SI unit in joules per coulomb (J/C),

$g \quad$ is the fraction of the initial kinetic energy of secondary electrons dissipated in air through radiative processes, but is negligible for $\mathrm{x}$ rays with energy less than $300 \mathrm{keV}$, and

$\Pi k_{i} \quad$ is the product of the correction factors to be applied to the free-air ionization chamber.

The physical constants used in the calculation of air kerma follow in Table 2. The calculation of air kerma involves some physical measurements of the primary standards, which are listed in Table 3. The dimensions of the chambers are used to determine the mass of air in which the ionization occurs.

Table 2. Physical constants used in the determination of air kerma

\begin{tabular}{lcc}
\hline $\begin{array}{l}\text { Physical } \\
\text { constant }\end{array}$ & Value & $\begin{array}{c}\text { Relative standard } \\
\text { uncertainty } \\
(\%)\end{array}$ \\
\hline$\rho_{\text {air }}$ a & $1.293 \mathrm{~kg} \cdot \mathrm{m}^{-3}$ & 0.01 \\
$W_{\text {air }} / e$ & $33.97 \mathrm{~J} \mathrm{C}$ & 0.15 \\
$1-g_{\text {air }}$ & 1.0000 & 0.01 \\
\hline
\end{tabular}

${ }^{\text {a }}$ Density of dry air at $T=273.15 \mathrm{~K}$ and $p=101325 \mathrm{~Pa}$.

\section{Characteristics of Air Kerma Standards}

\subsection{Description of Standards}

The measurement of the mass ionization current for the determination of air kerma is obtained at both NPL and NIST through the use of primary standard freeair ionization chambers. All four of the free-air ionization chambers used in the comparison are of the conventional parallel plate design. The diameter of the chamber aperture and the length of the collecting region define the mass of air in which the ionization is collected for this type of free-air ionization chamber. The NPL $50 \mathrm{kV}$ chamber is used to measure exposures and air kerma for $\mathrm{x}$ rays generated between $8 \mathrm{kV}$ and $50 \mathrm{kV}$. Reference [1] presents a detailed design description of the NPL chamber and a complete explanation of the correction factors. The NPL $50 \mathrm{kV}$ primary standard has a companion chamber that allows the direct measurement of the air attenuation correction. The air attenuation chamber is of similar construction as the 
Table 3. Dimensions of primary standard chambers used in the comparison

\begin{tabular}{lcccc}
\hline \hline Aperture diameter $(\mathrm{mm})$ & NIST Lamperti & NIST Ritz & NPL 50 kV & NPL 300 kV \\
\hline Air path length $(\mathrm{mm})$ & 39.18 & 127.39 & 89.2 & 493 \\
Aperture diameter $(\mathrm{mm})$ & 4.9943 & 10.0017 & 8.007 & 10.014 \\
Applied voltage $(\mathrm{V})$ & 1500 & $3000^{\mathrm{a}}$ & 1500 & 3000 \\
Collector length $(\mathrm{mm})$ & 10.135 & 70.03 & 19.827 & 100.258 \\
Volume $\left(\mathrm{mm}^{3}\right)$ & 198.55 & 5502.05 & 998.5 & 7896 \\
Plate separation $(\mathrm{mm})$ & 40 & 90 & 62.5 & 264 \\
\hline
\end{tabular}

${ }^{\mathrm{a}}$ An applied voltage of $5 \mathrm{kV}$ is routinely used at NIST for the Ritz chamber.

standard, but is fitted with two collector plates separated by the distance equal to the distance between the defining plane of the aperture and the center of the collecting volume of the primary standard. The NPL $300 \mathrm{kV}$ standard is a new chamber which has been designed for use with $\mathrm{x}$ rays generated between $40 \mathrm{kV}$ and $300 \mathrm{kV}$. The design has been described [2], but additional work on the scattered photon correction is continuing and a full report is being prepared. The NIST Lamperti chamber is designed for $\mathrm{x}$-ray exposure and air kerma measurements in the $10 \mathrm{kV}$ to $60 \mathrm{kV}$ region, but is generally used at NIST for the $10 \mathrm{kV}$ and $15 \mathrm{kV}$ qualities. The Lamperti chamber, described in detail in Ref. [3], utilizes a guard-ring system to maintain a uniform electric field. The NIST Ritz chamber, designed for $\mathrm{X}$-ray exposure and air kerma measurements between $20 \mathrm{kV}$ to $100 \mathrm{kV}$, uses a guard plate and guard strip system to diminish the distortion to the electric fields. The Ritz chamber is described in Ref. [4].

\subsection{Standard Correction Factors}

Although free-air chambers are designed to keep all corrections to the mass ionization current to a minimum, some corrections must be applied. The air attenuation correction, the largest of all the corrections, is the correction for the attenuation of the $x$ rays in the air between the defining plane of the chamber aperture and the center of the collecting volume. The air attenuation correction is expressed by

$$
k_{\mathrm{a}}=\exp (\mu L)
$$

where $\mu$ is the air-attenuation coefficient and $L$ is the air absorption length, the distance between the defining point of the chamber aperture and the center of the chamber volume.

All of the principal corrections for each chamber are listed in Tables 4 through Table 9. The air attenuation corrections are adjusted to the conditions of $293.15 \mathrm{~K}$ and $101.325 \mathrm{kPa}$. The humidity correction applied to all chambers, as well as its associated uncertainty, was taken from Ref. [5]. No investigation into the polarity effects was conducted for this comparison; previously determined polarity corrections were applied. The NPL $50 \mathrm{kV}$ chamber was designed to achieve no measurable front face penetration. The correction factors for wall transmission and ion loss for the NPL $300 \mathrm{kV}$ chamber are considered to be negligible at the energies used in this comparison. The Lamperti chamber corrections for wall transmission, $k_{\mathrm{p}}$ and aperture transmission, $k_{\mathrm{l}}$, are negligible with negligible uncertainties. The Ritz chamber is also considered to have negligible corrections for wall transmission and aperture transmission with relative standard uncertainties of $0.01 \%$ and $0.04 \%$, respectively. The ion recombination correction for the Ritz chamber was evaluated for an applied potential of $3 \mathrm{kV}$, less than the routinely used $5 \mathrm{kV}$.

\section{Comparison Procedure}

The collection of the charge measurements and the positioning of the primary standards was performed by an automated procedure for the majority of the reference radiation qualities used in this comparison. In the NPL low-energy range, the positioning of each standard is accomplished through the use of linear positioning motion controllers and verified manually with micrometers before and after each measurement series. Adjustments in positioning are made to the chamber position since the $\mathrm{x}$-ray tube is held in a fixed position. The typical measurement routine, used in the lowenergy NPL calibration facility, involved measuring the air attenuation correction with the NPL air attenuation chamber, measuring the charge with the NPL $50 \mathrm{kV}$ standard, followed by the NIST standard and completed with repeat measurements with the NPL standard and the NPL air attenuation chamber. The complete comparison measurement routine was conducted at least twice for each of the NIST standards.

The positioning routine used for this comparison in the NPL medium-energy facility was not ideal; the process was laborious and imprecise. Due to the size and weight of the NIST standard, the NPL normal calibration positioning procedures were unusable. Since the 
Table 4. Correction factors used for the Lamperti chamber for the NPL comparison

\begin{tabular}{|c|c|c|c|c|c|c|c|}
\hline \multirow[t]{2}{*}{ Correction factor } & \multicolumn{5}{|c|}{$\begin{array}{l}\text { Generating potential } \\
\qquad(\mathrm{kV})\end{array}$} & \multicolumn{2}{|c|}{$\begin{array}{l}\text { Relative standard uncertainty } \\
\qquad(\%)\end{array}$} \\
\hline & 10 & 11.5 & 14 & 16 & 20 & Type A & Type B \\
\hline Air attenuation $k_{\mathrm{a}}{ }^{\mathrm{a}}$ & 1.0721 & 1.0520 & 1.0342 & 1.0244 & 1.0169 & 0.05 & 0.23 \\
\hline Electron loss $k_{\mathrm{e}}$ & 1.000 & 1.000 & 1.000 & 1.000 & 1.000 & & 0.1 \\
\hline Field distortion $k_{\mathrm{d}}$ & 1.000 & 1.000 & 1.000 & 1.000 & 1.000 & & 0.2 \\
\hline Humidity $k_{\mathrm{h}}$ & 0.998 & 0.998 & 0.998 & 0.998 & 0.998 & & 0.06 \\
\hline Recombination $k_{\mathrm{s}}$ & 1.0001 & 1.0001 & 1.0001 & 1.0001 & 1.0001 & 0.04 & \\
\hline Photon scatter $k_{\mathrm{sc}}$ & 0.9960 & 0.9962 & 0.9963 & 0.9965 & 0.9966 & & 0.2 \\
\hline
\end{tabular}

a These are nominal values for $T=293.13 \mathrm{~K}$ and $p=101325 \mathrm{~Pa}$; each measurement is corrected using the air temperature and pressure measured at the collection time.

Table 5. Correction factors used for the Ritz chamber for tungsten reference radiation qualities used in the NPL comparison

\begin{tabular}{|c|c|c|c|c|c|c|c|c|c|}
\hline \multirow[t]{2}{*}{ Correction factor } & \multicolumn{7}{|c|}{$\begin{array}{l}\text { Generating potential } \\
\qquad(\mathrm{kV})\end{array}$} & \multicolumn{2}{|c|}{$\begin{array}{c}\text { Relative standard uncertainty } \\
\qquad(\%)\end{array}$} \\
\hline & 20 & 24 & 34 & 41 & 44 & 50 & 80 & Type A & Type B \\
\hline Air attenuation $k_{\mathrm{a}}{ }^{\mathrm{a}}$ & 1.0557 & 1.0371 & 1.0334 & 1.0253 & 1.0169 & 1.0113 & 1.0054 & 0.05 & 0.23 \\
\hline Aperture transmission $k_{1}$ & 1.0000 & 1.0000 & 1.0000 & 1.0000 & 1.0000 & 1.0000 & 1.0000 & & 0.04 \\
\hline Electron loss $k_{\mathrm{e}}$ & 1.0000 & 1.0000 & 1.0000 & 1.0000 & 1.0000 & 1.0000 & 1.0012 & & 0.10 \\
\hline Field distortion $k_{\mathrm{d}}$ & 1.000 & 1.000 & 1.000 & 1.000 & 1.000 & 1.000 & 1.000 & & 0.20 \\
\hline Humidity $k_{\mathrm{h}}$ & 0.998 & 0.998 & 0.998 & 0.998 & 0.998 & 0.998 & 0.998 & & 0.06 \\
\hline Recombinationb $k_{\mathrm{s}}$ & 1.0012 & 1.0010 & 1.0015 & 1.0016 & 1.0015 & $\begin{array}{l}1.0007 \\
1.0013^{\mathrm{c}}\end{array}$ & 1.0007 & 0.04 & \\
\hline Photon scatter $k_{\mathrm{sc}}$ & 0.9939 & 0.9944 & 0.9947 & 0.9950 & 0.9953 & 0.9956 & 0.9965 & & 0.20 \\
\hline Wall transmission $k_{\mathrm{p}}$ & 1.0000 & 1.0000 & 1.0000 & 1.0000 & 1.0000 & 1.0000 & 1.0000 & & 0.01 \\
\hline
\end{tabular}

${ }^{\text {a }}$ These are nominal values for $T=293.13 \mathrm{~K}$ and $p=101325 \mathrm{~Pa}$; each measurement is corrected using the air temperature and pressure measured at the collection time.

${ }^{\mathrm{b}}$ The recombination correction was evaluated for an applied potential of $3 \mathrm{kV}$.

${ }^{\mathrm{c}}$ Two values are shown for the recombination correction at $50 \mathrm{kV}$ due to the use of different air kerma rates .

Table 6. Correction factors used for the Ritz chamber for molybdenum reference radiation qualities used in the NPL comparison

\begin{tabular}{|c|c|c|c|c|}
\hline \multirow[t]{3}{*}{ Correction factor } & \multicolumn{2}{|c|}{$\begin{array}{l}\text { Generating potential } \\
\qquad(\mathrm{kV})\end{array}$} & \multicolumn{2}{|c|}{$\begin{array}{l}\text { Relative standard uncertainty } \\
\qquad(\%)\end{array}$} \\
\hline & 28 & 28 & Type A & Type B \\
\hline & \multicolumn{2}{|c|}{ with phantom } & & \\
\hline Air attenuation $k_{\mathrm{a}}{ }^{\mathrm{a}}$ & 1.0268 & 1.0219 & 0.05 & 0.23 \\
\hline Electron loss $k_{\mathrm{e}}$ & 1.0000 & 1.0000 & & 0.10 \\
\hline Field distortion $k_{\mathrm{d}}$ & 1.000 & 1.000 & & 0.20 \\
\hline Humidity $k_{\mathrm{h}}$ & 0.998 & 0.998 & & 0.06 \\
\hline Recombinationb $k_{\mathrm{s}}$ & 1.0004 & 1.0002 & 0.04 & \\
\hline Photon scatter $k_{\mathrm{sc}}$ & 0.9945 & 0.9952 & & 0.20 \\
\hline
\end{tabular}

a These are nominal values for $T=293.13 \mathrm{~K}$ and $p=101325 \mathrm{~Pa}$; each measurement is corrected using the air temperature and pressure measured at the collection time.

${ }^{b}$ The recombination correction was evaluated for an applied potential of $3 \mathrm{kV}$. 
Table 7. Correction factors for the NPL $50 \mathrm{kV}$ chamber for reference radiation qualities generated between $10 \mathrm{kV}$ and $20 \mathrm{kV}$

\begin{tabular}{|c|c|c|c|c|c|c|c|}
\hline \multirow[t]{2}{*}{ Correction factor } & \multicolumn{5}{|c|}{$\begin{array}{l}\text { Generating potential } \\
\qquad(\mathrm{kV})\end{array}$} & \multicolumn{2}{|c|}{$\begin{array}{l}\text { Relative standard uncertainty } \\
\qquad(\%)\end{array}$} \\
\hline & 10 & 11.5 & 14 & 16 & 20 & Type A & Type B \\
\hline Air attenuation $k_{\mathrm{a}}{ }^{\mathrm{a}}$ & 1.1749 & 1.1224 & 1.0804 & 1.0570 & 1.0398 & 0.05 & 0.23 \\
\hline Electron loss $k_{\mathrm{e}}$ & 1.0000 & 1.0000 & 1.0000 & 1.0000 & 1.0000 & & 0.006 \\
\hline Field distortion $k_{\mathrm{d}}$ & 1.0002 & 1.0002 & 1.0002 & 1.0002 & 1.0002 & & 0.012 \\
\hline Humidity $k_{\mathrm{h}}$ & 0.998 & 0.998 & 0.998 & 0.998 & 0.998 & & 0.058 \\
\hline Recombination $k_{\mathrm{s}}$ & 1.0004 & 1.0005 & 1.0005 & 1.0005 & 1.0008 & & 0.029 \\
\hline Photon scatter $k_{\mathrm{sc}}$ & 0.9949 & 0.9954 & 0.9959 & 0.9962 & 0.9967 & & 0.115 \\
\hline Polarity effect & 1.0004 & 1.0004 & 1.0004 & 1.0004 & 1.0004 & & 0.023 \\
\hline
\end{tabular}

${ }^{a}$ These are nominal values for $T=293.13 \mathrm{~K}$ and $p=101325 \mathrm{~Pa}$; each measurement is corrected using the air temperature and pressure measured at the collection time.

Table 8. Correction factors for the NPL $50 \mathrm{kV}$ chamber for reference radiation qualities generated between $24 \mathrm{kV}$ and $50 \mathrm{kV}$

\begin{tabular}{|c|c|c|c|c|c|c|c|c|c|}
\hline \multirow[t]{2}{*}{ Correction factor } & \multicolumn{7}{|c|}{$\begin{array}{l}\text { Generating potential } \\
\qquad(\mathrm{kV})\end{array}$} & \multicolumn{2}{|c|}{$\begin{array}{l}\text { Relative standard uncertainty } \\
\qquad(\%)\end{array}$} \\
\hline & 24 & 34 & 41 & 44 & 50 & Mo 28 & $\begin{array}{c}\text { Mo } 28 \\
\text { exit }\end{array}$ & Type A & Type B \\
\hline Air attenuation $k_{\mathrm{a}}{ }^{\mathrm{a}}$ & 1.0262 & 1.0233 & 1.0169 & 1.0121 & 1.0083 & 1.0231 & 1.0132 & 0.05 & 0.23 \\
\hline Electron loss $k_{\mathrm{e}}$ & 1.0000 & 1.0000 & 1.0000 & 1.0000 & 1.0000 & 1.0000 & 1.0000 & & 0.006 \\
\hline Field distortion $k_{\mathrm{d}}$ & 1.0002 & 1.0002 & 1.0002 & 1.0002 & 1.0002 & 1.0002 & 1.0002 & & 0.012 \\
\hline Humidity $k_{\mathrm{h}}$ & 0.998 & 0.998 & 0.998 & 0.998 & 0.998 & 0.998 & 0.998 & & 0.058 \\
\hline Recombination $k_{\mathrm{s}}$ & 1.0007 & 1.0012 & 1.0014 & 1.0013 & 1.0011 & 1.0000 & 1.0000 & & 0.029 \\
\hline Photon scatter $k_{\mathrm{sc}}$ & 0.9971 & 0.9973 & 0.9975 & 0.9977 & 0.9979 & 0.9972 & 0.9977 & & 0.115 \\
\hline Polarity effect & 1.0004 & 1.0004 & 1.0004 & 1.0004 & 1.0004 & 1.0004 & 1.0004 & & 0.023 \\
\hline
\end{tabular}

${ }^{\text {a }}$ These are nominal values for $T=293.13 \mathrm{~K}$ and $p=101325 \mathrm{~Pa}$; each measurement is corrected using the air temperature and pressure measured at the collection time.

Table 9. Correction factors for the NPL $300 \mathrm{kV}$ chamber for reference radiation qualities generated at $50 \mathrm{kV}$ and $80 \mathrm{kV}$

\begin{tabular}{lcccc}
\hline \hline Correction factor & \multicolumn{2}{c}{$\begin{array}{c}\text { Generating potential } \\
(\mathrm{kV})\end{array}$} & \multicolumn{2}{c}{$\begin{array}{c}\text { Relative standard uncertainty } \\
(\%)\end{array}$} \\
& 50 & 80 & Type A & Type B \\
\hline Air attenuation $k_{\mathrm{a}}{ }^{\mathrm{a}}$ & 1.0401 & 1.0202 & 0.20 & 0.23 \\
Electron loss $k_{\mathrm{e}}$ & 1.0000 & 1.0000 & & 0.006 \\
Field distortion $k_{\mathrm{d}}$ & 1.0003 & 1.0003 & & 0.012 \\
Humidity $k_{\mathrm{h}}$ & 0.998 & 0.998 & & 0.058 \\
Recombination $k_{\mathrm{s}}$ & 1.0004 & 1.0004 & 0.029 \\
Photon scatter $k_{\mathrm{sc}}$ & 0.9908 & 0.9926 & & 0.023 \\
Polarity effect & 1.0004 & 1.0004 & & 0.115 \\
\hline
\end{tabular}

${ }^{\text {a }}$ These are nominal values for $T=293.13 \mathrm{~K}$ and $p=101325 \mathrm{~Pa}$; each measurement is corrected using the air temperature and pressure measured at the collection time. 
NIST standard could not be positioned on the same alignment support structure as the NPL chamber, the NIST standard was independently positioned before each measurement. This resulted in a higher positioning uncertainty. The air temperature and pressure for each measurement was monitored using NPL instrumentation in both facilities.

\section{Measurement Uncertainties}

The individual uncertainty components, considered applicable to the comparison, are shown in Table 10 for all of the primary standards. The NIST uncertainties were evaluated according to Ref. [6]. In general, the uncertainties are representative of the uncertainties associated with routine air-kerma measurements at both institutions. The alignment and the charge measurement uncertainties for the NIST chambers result from the NPL alignment and charge collection methods and are different than those for air-kerma measurements conducted at NIST.

\section{Results and Conclusions}

The comparison results are shown in Tables 11 through Table 13 as the ratio of the corrected mass ionization current of the NIST primary standard to that of the NPL primary standard for each reference radiation used in the comparison. The Lamperti chamber response agreed with that of the NPL $50 \mathrm{kV}$ chamber response at the $0.5 \%$ level for the reference radiation qualities produced at $10 \mathrm{kV}$ and at the $\pm 0.1 \%$ level for the reference radiation qualities produced between $11.5 \mathrm{kV}$ and $20 \mathrm{kV}$. The Lamperti and the NPL chambers have good agreement, considering the uncertainty of this comparison. The Ritz chamber also compared favorably with the NPL $50 \mathrm{kV}$ chamber; agreement was found to be between $0.2 \%$ and $0.6 \%$ for both the tungsten and the molybdenum reference radiation qualities up to $50 \mathrm{kV}$. The Ritz chamber and the NPL $300 \mathrm{kV}$ chamber compared favorably, $0.1 \%$ and $0.6 \%$, for $50 \mathrm{kV}$ and $80 \mathrm{kV}$ respectively, despite the alignment difficulties. The Ritz chamber electron loss corrections and the photon scatter corrections are currently being reevaluated for new reference radiation qualities being developed at NIST. Slight changes to the corrections used for $80 \mathrm{kV}$ are expected; however the results for this comparison are based on the correction factors available at the time of the comparison.

Table 10. Applicable relative standard uncertainties for the indicated chamber for the 1998 NPL-NIST comparison, in percent

\begin{tabular}{|c|c|c|c|c|c|c|c|c|}
\hline \multirow{2}{*}{$\begin{array}{l}\text { Source of uncertainty } \\
\text { Combined relative standard }\end{array}$} & \multicolumn{2}{|c|}{ NIST Lamperti } & \multicolumn{2}{|c|}{ NIST Ritz } & \multicolumn{2}{|c|}{ NPL $50 \mathrm{kV}$} & \multicolumn{2}{|c|}{ NPL $300 \mathrm{kV}$} \\
\hline & Type A & Type B & Type A & Type B & Type A & Type B & Type A & Type B \\
\hline Ionization current & 0.100 & 0.017 & 0.100 & 0.017 & 0.100 & 0.017 & 0.100 & 0.017 \\
\hline Volume & 0.04 & 0.01 & 0.04 & 0.01 & & 0.155 & & 0.155 \\
\hline Positioning & & 0.10 & & 0.10 & & 0.10 & & \\
\hline Correction factors (excluding $k_{\mathrm{h}}$ ) & 0.064 & 0.326 & 0.064 & 0.385 & 0.05 & 0.267 & 0.2 & 0.238 \\
\hline Humidity $k_{\mathrm{h}}$ & & 0.06 & & 0.06 & & 0.06 & & 0.06 \\
\hline Physical constants & & 0.15 & & 0.15 & & 0.15 & & 0.15 \\
\hline Quadratic sum & 0.13 & 0.38 & 0.13 & 0.43 & 0.11 & 0.36 & 0.22 & 0.33 \\
\hline $\begin{array}{l}\text { Combined relative standard } \\
\text { uncertainty for the } \\
\text { measurement of uncertainty }\end{array}$ & \multicolumn{2}{|c|}{0.40} & \multicolumn{2}{|c|}{0.45} & \multicolumn{2}{|c|}{0.38} & \multicolumn{2}{|c|}{0.40} \\
\hline
\end{tabular}

Table 11. Comparison of Lamperti chamber to the $50 \mathrm{kV}$ NPL standard

\begin{tabular}{cccc}
\hline \hline $\begin{array}{c}\text { NPL reference } \\
\text { number }\end{array}$ & $\begin{array}{c}\text { Generating potential } \\
(\mathrm{kV})\end{array}$ & $\begin{array}{c}\text { Half-value layer } \\
(\mathrm{mm} \mathrm{Al})\end{array}$ & $\begin{array}{c}\text { Ratio of the NIST } \\
\text { to the NPL standard } \\
\text { chamber response }\end{array}$ \\
\hline 2.4 .2 & 10 & 0.036 & 0.9951 \\
2.4 .3 & 11.5 & 0.05 & 1.0006 \\
2.4 .4 & 14 & 0.07 & 0.9996 \\
2.4 .5 & 16 & 0.1 & 0.9995 \\
2.4 .6 & 20 & 0.15 & 0.9992 \\
\hline
\end{tabular}


Table 12. Comparison of the Ritz chamber to the NPL $50 \mathrm{kV}$ standard

\begin{tabular}{lccc}
\hline $\begin{array}{c}\text { NPL reference } \\
\text { number }\end{array}$ & $\begin{array}{c}\text { Generating } \\
\text { potential } \\
(\mathrm{kV})\end{array}$ & $\begin{array}{c}\text { Half-value layer } \\
(\mathrm{mm} \mathrm{Al})\end{array}$ & $\begin{array}{c}\text { Ratio of the NIST } \\
\text { to the NPL standard } \\
\text { chamber response }\end{array}$ \\
\hline 2.4 .6 & 20 & 0.15 & 0.9977 \\
2.4 .7 & 24 & 0.25 & 0.9978 \\
2.4 .8 & 34 & 0.35 & 0.9989 \\
2.4 .9 & 41 & 0.5 & 0.9973 \\
2.4 .10 & 44 & 0.7 & 0.9983 \\
2.4 .11 & 50 & 1.0 & 0.9983 \\
Mo 28 & 28 & 0.30 & 0.9958 \\
Mo 28 Exit & 28 & 0.62 & 0.9937 \\
\hline
\end{tabular}

Table 13. Comparison of the Ritz chamber to the NPL $50 \mathrm{kV}$ standard

\begin{tabular}{cccc}
\hline $\begin{array}{c}\text { NPL reference } \\
\text { number }\end{array}$ & $\begin{array}{c}\text { Generating } \\
\text { potential } \\
(\mathrm{kV})\end{array}$ & $\begin{array}{c}\text { Half-value layer } \\
(\mathrm{mm} \mathrm{Al})\end{array}$ & $\begin{array}{c}\text { Ratio of the NIST } \\
\text { to the NPL standard } \\
\text { chamber response }\end{array}$ \\
\hline 2.2 .1 & 50 & 1.0 & 0.9987 \\
RQR6 & 80 & 2.9 & 0.9941 \\
\hline
\end{tabular}

\section{References}

[1] A. R. S. Marsh and T. T. Williams, $50 \mathrm{kV}$ Primary Standard of Exposure-1978 Design Of Free-Air Chamber, RS (EXT)54, National Physical Laboratory Report, Teddington, Middlesex, UK, April 1982.

[2] J. A. Palmer, S. Duane, D. R. Shipley, and C. J. Moretti, The Design and Construction of a New Primary Standard Free Air Chamber for Medium Energy X-rays, Medical and Biological Engineering and Computing, 35, 1086 (1997)

[3] P. J. Lamperti and H. O. Wyckoff, NBS Free-Air Chamber for Measurement of 10 to $60 \mathrm{kV}$ X Rays, J. Res. Natl. Bur. Stand. (U.S.) 69C, 39-46 (1965).

[4] V. H. Ritz, Design of Free-Air Ionization Chambers for the Soft X-Ray Region (20-100 kV), Radiology, 73(6), 911-922 (1959).

[5] International Commission on Radiation Units and Measurements, Average Energy Required to Produce an Ion Pair, ICRU 31, Washington, D.C. (1979).

[6] B. N. Taylor and C. E. Kuyatt, Guidelines for Evaluating and Expressing the Uncertainty of NIST Measurement Results, 1994 Edition, NIST Technical Note 1297, September 1994.

Please note corrections to the electronic version of this article

Tables 11, 12, and 13 were incorrect in the original print and electronic versions of this article. They are correct here, and an Erratum will be published to correct the printed Journal.
About the authors: Michelle O'Brien and Paul Lamperti are physicists in the Ionizing Radiation Division, Dosimetry and Interaction Group of the NIST Physics Laboratory. Tudor Williams and Thorsten Sander are physicists in the Centre for Ionising Radiation Metrology at the National Physical Laboratory, United Kingdom. The National Bureau of Standards and Technology is an agency of the Technology Administration, U.S. Department of Commerce. 